\title{
Trigeminal neuralgia linked to demyelination in multiple sclerosis
}

Trigeminal neuralgia (TN), a facial pain disorder caused by irritation of the trigeminal nerve, is known to occur in many people with multiple sclerosis (MS). A new study published in Acta Neurologica Scandinavica has delved further into the relationship between these two conditions, and the results suggest an association between $\mathrm{TN}$ and the presence of demyelinating lesions in the vicinity of the trigeminal ganglia.

"We wanted to understand whether TN is a syndrome appearing in the late stage of MS, when MS is generally characterized by slowly progressing degeneration of the brain, or whether it could be the first episode of MS, thus linked more closely to episodes of systemically driven neuroinflammation," explains corresponding author Sini Laakso.

The new study included 2,575 patients with MS from the
Finnish MS register and 2,008 patients with TN who were identified from a hospital administrative database. From the total cohort, 55 individuals had both MS and $\mathrm{TN}$, and the incidence of $\mathrm{TN}$ was found to be 15-fold higher among patients with MS than in

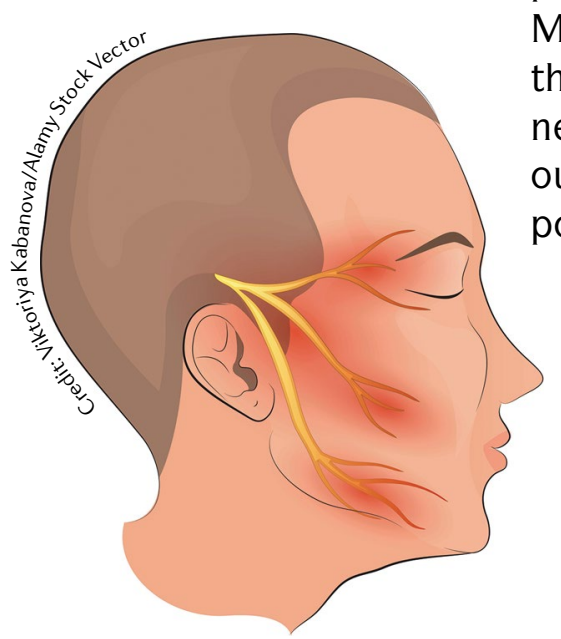

the general neurological outpatient population.

Laakso and colleagues obtained head MRI scans from 41 of the patients who had both MS and TN. In 26 (63\%) of these individuals, demyelinating lesions were found in close proximity to the trigeminal ganglia. Most of the patients with MS who developed TN had relapsing-remitting MS, in which inflammation and demyelination are the predominant pathogenetic mechanisms.

"Together, these findings suggest that there is a strong association between TN and MS, and that if an acute demyelinating lesion is identified close to the trigeminal nucleus, the onset of TN should be considered a relapse of MS," comments Laakso. "A prospective study is now needed to investigate the presence of acute demyelinating lesions at the time of onset of TN."

Heather Wood

ORIGINAL ARTICLE Laakso, S. M. et al. Trigeminal neuralgia in multiple sclerosis: prevalence and association with demyelination. Acta Neurol. Scand. https://doi.org/10.1111/ane.13243 (2020)

\section{Mechanistic insight into the spread of tau pathology}

A molecular mediator of pathological tau propagation has been identified in a new study published in Nature. The work shows that LDL receptor-related protein 1 (LRP1) is central to the spread of tau and could be a therapeutic target in several neurodegenerative diseases.

Tau protein aggregates are a pathological feature of multiple neurodegenerative disorders - known as tauopathies - including Alzheimer disease, frontotemporal dementia and chronic traumatic encephalopathy. Propagation of tau through the brain is associated with disease progression, but the molecular mechanisms of its spread from cell to cell are unclear. "The problem of tau uptake and spread is one of the key missing pieces of data needed to understand the pathology of tauopathies," explains Kenneth Kosik, who led the new study.
Cᄃ

\section{Blocking LRP1}

was a powerful

means to block

tau spread

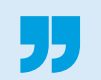

Kosik and colleagues previously showed that heparan sulfate proteoglycans (HSPGs) were involved in tau propagation. LRP1 can work with HSPGs to mediate amyloid- $\beta$ uptake, so the team investigated whether LDL receptors are also involved in tau uptake.

The team first used CRISPR interference to suppress expression of seven LDL receptors in $\mathrm{H} 4$ neuroglioma cells. LRP1 suppression blocked uptake of tau monomers and oligomers, but suppression of the other LDL receptors did not affect tau uptake. Use of CRISPR interference to knock down LRP1 in human induced pluripotent stem cell-derived neurons reduced uptake of tau into these cells.

Kosik and colleagues also investigated the role of LRP1 in tau propagation in the brains of mice.
Targeted neuronal knockdown of LRP1 in a mouse model of tau spread demonstrated that absence of this receptor substantially reduced the spread of tau pathology. "Blocking LRP1 was a powerful means to block tau spread," concludes Kosik.

The findings raise the possibility of targeting LRP1 to prevent the propagation of tau pathology and, consequently, the progression of tauopathies. However, Kosik warns that this approach would not be as simple as it seems. "Complete blockade of LRP1 is not a good strategy because it is important for other key functions as well as tau uptake," he explains. "The way to move forward and translate these findings to the clinical setting is to zero in on how the receptor mediates tau uptake with its other relevant interactions, such as that with apolipoprotein E."

ORIGINAL ARTICLE Rauch, J. N. et al. LRP1 is a master regulator of tau uptake and spread. Nature 580, 381-385 (2020) RELATED ARTICLE Peng, C. et al. Protein transmission in neurodegenerative disease. Nat. Rev. Neurol. 16, 199-212 (2020) 